
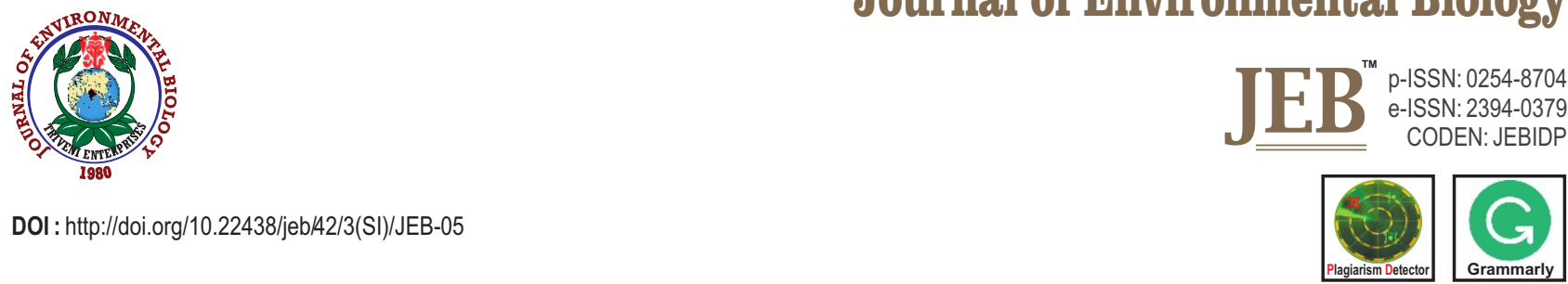

\title{
Allelopathic screening of Malaysian noxious weeds and several medicinal plants as potential alleloherbicides
}

\author{
M.S. Ishak', M.B. Nurul Ain ${ }^{1}$, I. Sahid' and K. Mardiana-Jansar ${ }^{1 *}$ \\ ${ }^{1}$ Department of Earth Science and Environment, Faculty of Science and Technology, Universiti Kebangsaan Malaysia, Bangi, 43600, Malaysia \\ *Corresponding Author Email : mardiana@ukm.edu.my
}

\section{Abstract}

Aim: This research was conducted to assess the allelopathic potential of 100 plants species, including noxious weeds and medicinal plants of Malaysia as donor plant on the growth of Lactuca sativa (lettuce) as the recipient plant. This study was also carried out to justify common weeds found in Malaysia to act as a natural herbicide for weed management through allelopathy phenomenon.

Methodology: Weed and medicinal plant species commonly found in Malaysia were identified according to their morphological characteristics. Sandwich method was carried out to determine the allelopathic potential of weed species through leaf litter leachate mechanism. On the other hand, dish pack method was conducted to discover the allelopathic potential of weed species through volatilization pathway.

Results: 100 weed species and medicinal plants commonly found in Malaysia inhibited the radicle and hypocotyl growth of $L$. sativa by both sandwich method and dish pack method. Among 100 weed species, Passiflora foetida showed the maximum inhibitory effects on $L$. sativa growth in both sandwich and dish pack method. The highest amount of $P$. foetida leaf litter $(50 \mathrm{mg})$, completely inhibited the growth of radicle and hypocotyl of $L$. sativa. Volatilization of weed and medicinal plants leaf litter inhibited the growth of $L$. sativa with increasing distances of lettuce seed with the weed and medicinal plants leaf litter.

Interpretation: The findings of this study provides a base line for identifying plants with allelopathic properties for controlling weed propagation.

Key words: Allelopathic potential, Allelopathy, Leaf litter leachate, Noxious weed, Volatilization

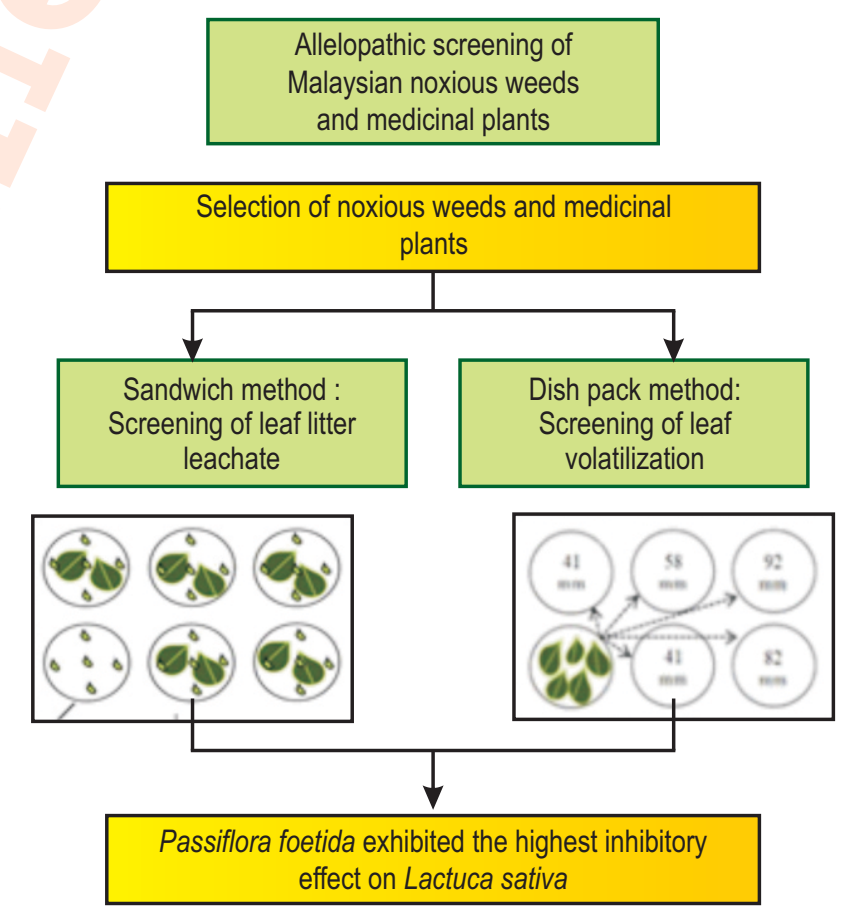

How to cite : Ishak, M.S., M.B. Nurul Ain, I. Sahid and K. Mardiana-Jansar: Allelopathic screening of Malaysian noxious weeds and several medicinal plants as potential alleloherbicides. J. Environ. Biol., 42, 762-774 (2021). 


\section{Introduction}

Interference of weeds in the growth of crops has been one of the contributing factors to low productivity of crops in Malaysia (Dilipkumar et al., 2017). The declining pattern of agricultural productivity is due to competition between the weeds and crops grown (Daehler et al., 2004). According to Sahid and Chan (2000), the competition between weeds and crops is mainly to obtain available nutrients, moisture, space and resources, which simultaneously reduces the productivity and quality of crops. Various efforts have been made to eradicate the problem of weed propagation, including utilization of more synonymous synthetic herbicides among farmers which reduces time and energy consumption. However, prolonged use of synthetic herbicides is risky as it poses direct or indirect harmful effect on the environment and human health (Aktar et al., 2009; Jaishankar et al., 2014).

The term allelopathy is defined as a biochemical interaction involving both inhibition and stimulation or one of them through the production of chemicals from one plant including microorganisms on another plant (Asaduzzaman et al., 2010). In general, allelopathy is a natural phenomenon that in certain types of plants by releasing allelochemical in their immediate environment through root exudates, litter leachate, volatilization, or decaying of tissue residues (Qin et al., 2006). According to de Albuquerque (2010), plants that possess allelopathic properties and release allelochemicals are known as donor plants while plants that are affected by allelochemical compounds secreted by other plants are known as recipient plants. Donor plants involve selected species of noxious weeds consisting of variety of families and morphological features, while Lactuca sativa (lettuce) seeds are used as recipient plants.

Volatilization is one way of spreading allelochemicals through allelopathic action that involves the release of volatile chemical compounds in the environment. Naturally, released chemicals are absorbed by the surrounding organisms by entering the soil structure and absorbed by the roots in the form of vapor released during condensation process. Dish pack method developed by Fujii et al. (2005) is one of the bioassay method for determining allelochemical volatilization to be carried out in laboratory. Generally, this method is used to analyze volatile allelochemicals where dried leaves of donor plants are placed in one of the holes known as source wells in a six-well multidish to note the effect of donor plant on the growth of bioassay species. This method is used as an approach to conform to the natural volatilization process. Fujii et al. (2005) observed that Cleome spinosa plant species inhibited $100 \%$ elongation of root and shoot of lettuce seedlings representing as bioassay species.

Litter leachate is also one of the pathways of allelopathic dissemination through the action of allelopathy that occurs when dissolved allelopathic compounds from the plants' aerial part (shoots) and released through rainwater, dew or drainage into the soil or to other plant surfaces (Chick and Keilbaso, 1998). Sandwich method was developed by Fujii et al. (2004) to determine the effects of leachate in laboratory, which showed the natural process of allelochemical emission from litter leachate to the environment. According to Fujii et al. (2004), sandwich method is used to study the inhibition effect of leaf debris placed in two layers of agar as a medium of dispersion. Shiraishi et al. (2002) used sandwich method to screen allelopathic activity through leaf litter leachate from 71 plant species and found that at least seven plant species inhibited the growth of lettuce seeds above 50\%. In addition, Morikawa et al. (2012) evaluated the allelopathic activity of several plant species in Peru and found that families Asteraceae, Anacardiaceae, Fabaceae, Solanaceae and Bombaceae inhibited the root growth of lettuce more than $80 \%$.

Generally, allelopathic plants in the current study in Malaysia involved only certain plant species that were found to have allelopathic potential in controlling weeds. Ishak et al. (2016) reported that Leucaena leucocephala tree interfered with the germination and growth of Ageratum conyzoides, Tridax procumbens and Emilia sonchifolia. A study by Ismail et al. (2015) involving the allelopathic assessment of weed species known as Pennisetum purpureum as a donor plant on the germination and growth of Eleusine indica. Moreover, Samedani et al. (2015) studied the effects of weed species of Axonopus compressus to control the growth of important weeds in oil palm plantation. In view of the above, this study aimed to evaluate the allelopathic potential of 100 plant species including noxious weeds and several medicinal plants commonly found in Malaysia through volatilization and litter leachate mode of action towards the growth of $L$. sativa.

\section{Materials and Methods}

Plants material: Fresh leaves of 100 species of common weeds and medicinal plants that served as donor plants found in Malaysia were collected from several places around Bangi, Selangor, Klang, Selangor, Sungai Rambai, Melaka, Pekan, Pahang and also around Telok Intan, Perak. Table 1 shows a list of weeds collected and were divided into four categories based on their morphological features namely broad leaves; $n=54$, grasses; $n=24$, sedges; $n=6$, and ferns; $n=16$. The weed samples were washed and dried in an oven at $60^{\circ} \mathrm{C}$ for $24 \mathrm{hr}$. Leaf samples were cut into $3.0 \times 3.0 \mathrm{~mm}$ pieces with scissors, stored in sealed plastic and labeled with their respective weed species and date of sample collection. Seeds of Lactuca sativa (lettuce) purchased from Takii Seed Co. Ltd., Kyoto, Japan were used as a recipient plant in this study due to its easy-to-grow potential and sensitivity to the environmental changes (Fujii et al., 2003).

Identification of weed species: The identification of 100 species of noxious weeds and medicinal plants in this study were determined by identifying and comparing the overall morphology 
Table 1: Weed species collected from various location in Malaysia

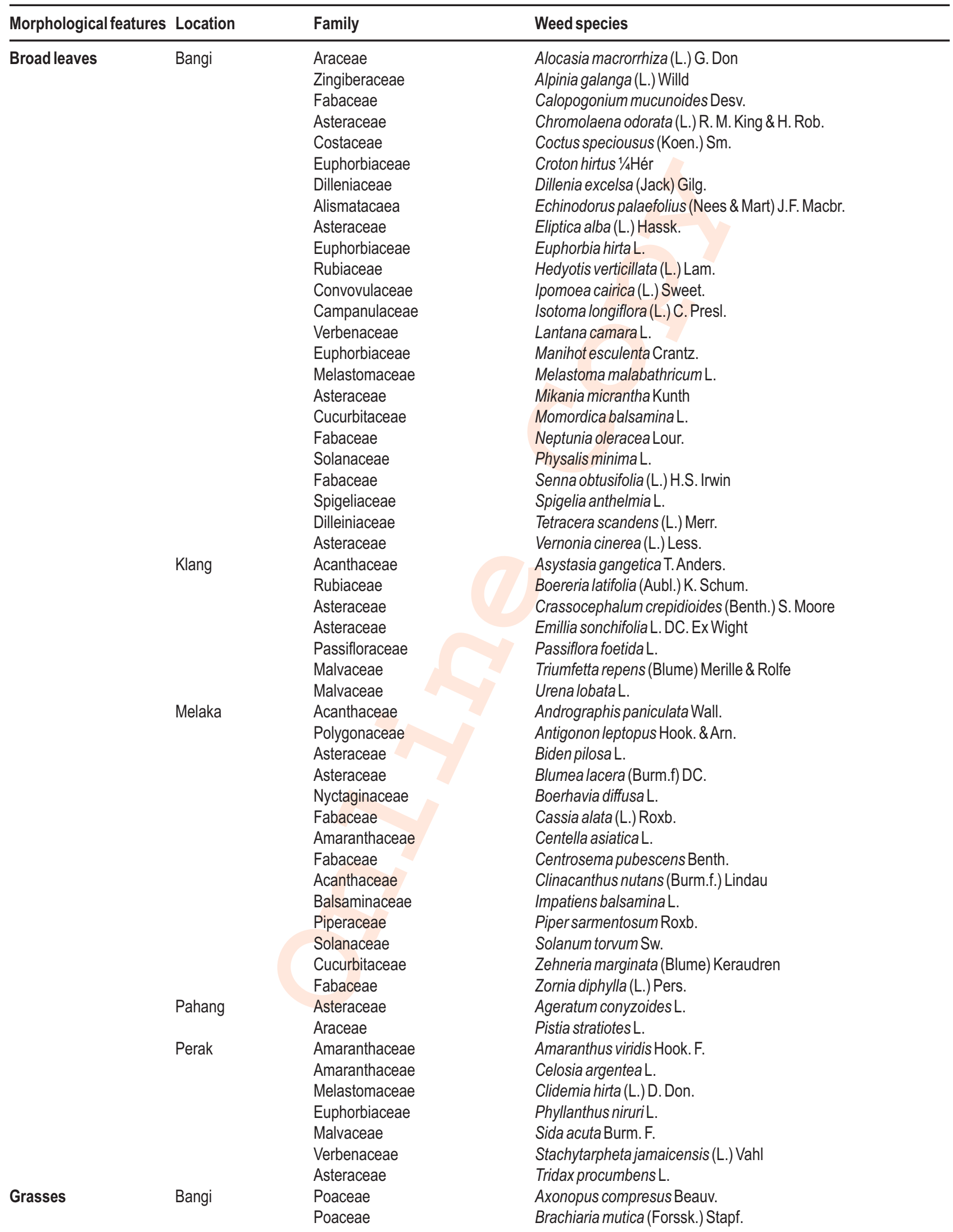




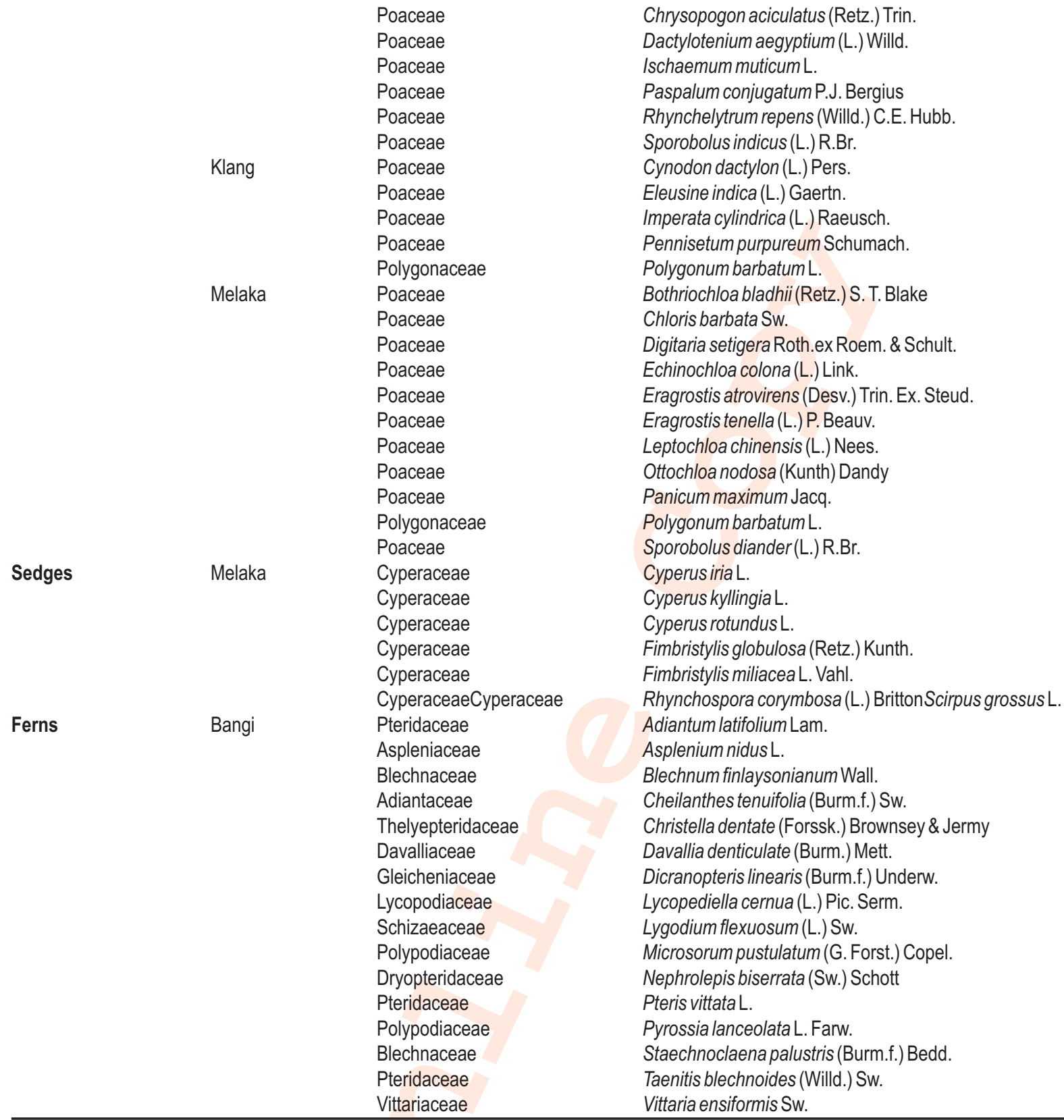

aspects of the plant such as flower, leaves, fruits, roots, stems and the nature of plants in their habitat. The species were then divided into four categories based on their morphological features namely broad leaves, grasses, sedges and ferns.

Sandwich method: A $0 \mathrm{mg}, 10 \mathrm{mg}$ and $50 \mathrm{mg}$ of different leaf litters were used in sandwich method in three replicates $(n=3)$. Leaf samples placed in $35 \mathrm{~mm}$ x $18 \mathrm{~mm}$ multidish (Nalga Nunc. International, Tokyo). A $5 \mathrm{ml}$ of autoclaved agar growth medium (Nacalai Tesque, Kyoto, Japan) (for $15 \mathrm{~min}$ at $121^{\circ} \mathrm{C}$ at $121 \mathrm{kPa}$ ) was then added to each leaf litter sample as first agar layer and left until the agar solidified at room temperature. Subsequently, another $5 \mathrm{ml}$ of growth medium was added to each multidish as a second layer and left to solid by for $5 \mathrm{~min}$. A total of $10 \mathrm{ml}$ of agar media for growth was poured into a multidish with no testable leaf samples being used as control. After the agar medium layer solidified, five seeds of lettuce are added upright onto the surface of agar. Each multidish was labeled with the name of respective weed species and sealed with adhesive tape. Each of the multiwell plates were then incubated at $20^{\circ} \mathrm{C}$ ( $24 \mathrm{hr}$ photoperiod) for four days. The length of hypocotyl and radicle of lettuce seeds were measured and recorded after four days. The collected data were used to calculate the percentage of growth of lettuce seeds at both leaf volume levels (10 $\mathrm{mg}$ and $50 \mathrm{mg}$ ) compared to the 
percentage of growth from control.

Dish pack method: Six well multidish plates $35 \mathrm{~mm}$ x $18 \mathrm{~mm}$ were used for dish pack method where the first hole of the multiplate was filled with $200 \mathrm{mg}$ of leaf sample and named as source well. Meanwhile, the remaining five mutildish holes were dispatched with Whatman No. 6 filter paper and three replicates $(n=3)$ were used for each weed species. Approximately, $0.7 \mathrm{ml}$ of distilled water was added onto the filter paper. Based on six well multidish plates used, the distances between the source well and other wells were $41,58,82$ and $92 \mathrm{~mm}$, respectively. A total of five lettuce seeds as a bioassay plant were added to the surface of filter paper at a uniform distance. For control treatment, no source well was employed; the first well of the multidish did not contain any weed leaf samples. Next, each of the multidish plates were labeled with their respective weed species and tightly sealed with plastic tape to prevent the emission of volatile matter from the leaf samples. Then, each multidish plate was stored at $20^{\circ} \mathrm{C}(24 \mathrm{hr}$ photoperiod) for 4 days. Hypocotyl and radicle length of lettuce seedlings were measured and recorded after 4 days. The data collected were used to calculate the growth percentage of lettuce seeds at four different distances and compared with the growth percentage of control plants.

Statistical analysis: The elongation of hypocotyl and radicle length of lettuce seedlings were measured and the percentage was calculated by comparison to the control readings. The experimental design was carried out using a complete randomized design with each concentration and species involving three replicates. The mean and standard deviation were calculated using Microsoft Excel 2013. To assess the activity and allelopathic effects of each weed species studied, the criterion was translated into SD (SDV) value using the formula [(100\% Min) - (0.5/1.0/1.5 (SD)] (Fujii et al., 2003, 2004). SDV value was used to identify the potential of 100 weed species studied as the best allelopathic plant that can inhibition the growth of lettuce.

\section{Results and Discussion}

Allelopathic potential screening using sandwich method: Sandwich method presents preliminary information as a benchmark in which it is closely related to the release of chemical compounds involved in natural allelopathy phenomena through leachate mechanism (Fujii et al., 2003; Shinwari et al., 2013). Table 2 shows the percent inhibition on radicle and hypocotyl growth of lettuce as a bioassay species when treated with two different mass of leaf debris, i.e., $10 \mathrm{mg}$ and $50 \mathrm{mg}$ of 100 dried samples of weed species having different morphological characteristics. Weed species listed in Table 2 were divided by the level of allelopathic activity ranging from the highest to the lowest percentage and classified into five categories (> 80\%, 60-79\%, $40-59 \%, 20-39 \%$ and $0-19 \%$ ) by following the method of Fujii et al. (2003; 2004).
The perusal of data showed that leaf extract of 100 weed and medicinal plant species inhibited the growth of radicle and hypocotyl of lettuce with the value ranging from 19.26 to $85.41 \%$ when tested with $10 \mathrm{mg}$ of dried sample for each weed species. Based on the screening through sandwich method, four species of weeds including Passiflora foetida, Piper sarmentosum, Clidemia hirta and Dicranopteris linearis strongly inhibited the root of lettuce seedlings by more than $80 \%$. On the contrary, only three weed species (Lygodium flexuosum, Christella dentata and Stachytarpheta jamaicensis) caused less inhibition of root seedlings of lettuce when sown with $10 \mathrm{mg}$ of weed leaf debris. Higher amount of leaf litter leachate $(50 \mathrm{mg}$ ) applied to all test weeds caused $34-100 \%$ higher inhibition on the root growth of lettuce compared to the application of $10 \mathrm{mg}$ weed leaf extract. These results corroborate with study from Fujii et al. (2003) who demonstrated that inhibition percentage of lettuce shoots increased with the increasing amount of leaf extract from several medicinal plants species such as Eucharis grandifolia, Annona cherimola and Emblica pectinata. Morikawa et al. (2012) had screened 170 plant species in Peru using sandwich method and found that $50 \mathrm{mg}$ of leaf debris from Aristeguieta ballii, Diplostephium foliosissimum and Spondias mombin resulted in $90 \%$ inhibition of root and shoot growth of lettuce seedlings. Similarly, Ali et al. (2019) found that the radicle growth of lettuce seedlings were inhibited when treated with $50 \mathrm{mg}$ of leaf debris of 196 medicinal plants species in Pakistan. From this study, it can be concluded that the leaf litter leachate of $P$. foetida showed the highest allelopathic effect among 100 weed species tested. Allelochemicals accumulated in $P$. foetida leaves can inhibit the growth and production of target plants by interfering with the plant physiology, directly or indirectly (Chou, 1999; Harper and Balke, 1981; Reigosa etal., 1999).

Allelopathic potential screening using dish pack method: Tables 3 and 4 shows the percent growth inhibition of root and shoots of lettuce treated with $200 \mathrm{mg}$ leaf litter from 100 weed species of different families having different morphological characteristics. This study was conducted by following the method of Fujii et al. (2005) to evaluate the effect of allelopathy on the growth of bioassay species by releasing chemical compounds through volatilization. In dish pack method, the position between the source well (contain weed leaf litter) and the position of lettuce seed (as the recipient plant) was tested at four different distances of $41 \mathrm{~mm}, 58 \mathrm{~mm}, 82 \mathrm{~mm}$ and $92 \mathrm{~mm}$. The control was multidish without weed and leaf litter of medicinal plants.

Inhibition of radicle and hypocotyl growth of lettuce were significant $(p<0.05)$ by leaf volatilization of different weed species at different distances. Volatilization of leaf litter for 100 weed species studied at the nearest distance of $41 \mathrm{~mm}$ inhibited the growth of lettuce radicle by 8.6 to $100 \%$. Percentage inhibition of radicle and hypocotyl of lettuce seed showed positive (inhibition) or negative value (growth promotion). After treating 
Table 2 : Allelopathic activity (\% inhibition of lettuce seed growth) based on 100 common weed and medicinal plant species found in Malaysia using sandwich method

\begin{tabular}{|c|c|c|c|c|c|}
\hline \multirow[t]{3}{*}{ Species name } & \multirow{3}{*}{$\begin{array}{l}\text { Morphological } \\
\text { characteristics }\end{array}$} & \multicolumn{2}{|c|}{ Debris mass $10 \mathrm{mg}$} & \multicolumn{2}{|c|}{ Debris mass $50 \mathrm{mg}$} \\
\hline & & \multicolumn{4}{|c|}{ Inhibition percentage (\%) } \\
\hline & & Radicle( $( \pm)$ & Hypocotyl( $( \pm)$ & Radicle( \pm ) & Hypocotyl( $( \pm)$ \\
\hline Passiflora foetida & Broad leaves & 85.41 & 23.92 & 100.00 & 100.00 \\
\hline Piper sarmentosum & Broad leaves & 87.93 & 77.65 & 96.59 & 90.20 \\
\hline Ageratum conyzoides & Broad leaves & 76.81 & 46.67 & 94.44 & 54.64 \\
\hline Zehneria marginata & Broad leaves & 68.59 & 44.84 & 93.93 & 70.46 \\
\hline Asplenium nidus & Fern & 56.37 & 13.46 & 93.93 & 42.09 \\
\hline Melastoma malabathricum & Broad leaves & 64.81 & 38.43 & 93.11 & 77.78 \\
\hline Clidemia hirta & Broad leaves & 81.56 & 52.29 & 93.04 & 86.14 \\
\hline Momordica balsamina & Broad leaves & 78.37 & 52.29 & 91.78 & 82.75 \\
\hline Centrosema pubescens & Broad leaves & 77.04 & 39.08 & 91.26 & 70.07 \\
\hline Rhynchelytrum repens & Grass & 65.19 & 25.62 & 91.11 & 61.31 \\
\hline Croton hirtus & Broad leaves & 75.11 & 43.79 & 91.04 & 72.81 \\
\hline Eliptica alba & Broad leaves & 71.93 & 29.41 & 90.81 & 57.91 \\
\hline Digitaria setigera & Grass & 59.11 & 29.28 & 88.67 & 72.03 \\
\hline Tridax procumbens & Broad leaves & 56.59 & 14.89 & 88 & 64.97 \\
\hline Clinacanthus nutans & Broad leaves & 62.74 & 30.33 & 87.85 & 63.79 \\
\hline Neptunia oleracea & Broad leaves & 66.96 & 41.96 & 87.70 & 68.63 \\
\hline Amaranthus viridis & Broad leaves & 66.96 & 4.84 & 87.33 & 67.58 \\
\hline Zornia diphylla & Broad leaves & 73.41 & 43.01 & 87.11 & 58.56 \\
\hline Cynodon dactylon & Grass & 73.63 & 20.78 & 86.44 & 48.37 \\
\hline Adiantum latifolium & Fern & 71.11 & 0.26 & 86.33 & 50.39 \\
\hline Fimbristylis miliacea & Sedge & 56.59 & 37.39 & 86.22 & 58.82 \\
\hline Dicranopteris linearis & Fern & 81.70 & 53.73 & 86.07 & 59.08 \\
\hline Solanum torvum & Broad leaves & 56.89 & 23.53 & 85.70 & 72.29 \\
\hline Alocasia macrorrhiza & Broad leaves & 59.78 & 22.35 & 84.96 & 47.71 \\
\hline Hedyotis verticillata & Broad leaves & 63.63 & 34.51 & 84.52 & 53.13 \\
\hline Ipomoea cairica & Broad leaves & 64.22 & 25.88 & 84.37 & 64.84 \\
\hline Pistia stratiotes & Broad leaves & 56.30 & 38.95 & 84.15 & 63.53 \\
\hline Echinochloa colona & Grass & 64.96 & 3.92 & 84.00 & 23.92 \\
\hline Asystasia gangentica & Broad leaves & 78.07 & 41.44 & 83.48 & 47.06 \\
\hline Polygonum barbatum & Grass & 64.30 & 28.10 & 83.26 & 58.69 \\
\hline Andrographis paniculata & Broad leaves & 64.37 & 56.21 & 83.11 & 72.55 \\
\hline Calopogonium mucunoides & Broad leaves & 63.11 & 5.49 & 83.04 & 29.80 \\
\hline Chromolaena odorata & Broad leaves & 79.41 & 51.90 & 82.89 & 66.27 \\
\hline Blechnum finlaysonianum & Fern & 22.81 & 2.88 & 82.59 & 25.49 \\
\hline Triumfetta repens & Broad leaves & 66.52 & -15.56 & 82.44 & 31.76 \\
\hline Sporobolus indicus & Grass & 61.33 & 31.24 & 81.93 & 37.12 \\
\hline Isotoma longiflora & Broad leaves & 58.52 & 26.14 & 81.85 & 52.68 \\
\hline Microsorum pustulatum & Fern & 41.26 & 18.30 & 81.78 & 64.18 \\
\hline Chloris barbata & Grass & 63.93 & 20.26 & 81.78 & 52.16 \\
\hline Eragrostis atrovirens & Grass & 47.93 & 24.71 & 81.41 & 58.95 \\
\hline Dactylotenium aegyptium & Grass & 56.81 & 33.45 & 81.04 & 59.74 \\
\hline Senna obtusifolia & Broad leaves & 73.63 & 54.90 & 80.89 & 83.46 \\
\hline Phyllanthus niruri & Broad leaves & 37.48 & 37.48 & 80.74 & 29.54 \\
\hline Boereria latifolia & Broad leaves & 66.22 & 20.39 & 80.52 & 42.88 \\
\hline Sporobolus diander & Grass & 54.30 & 26.93 & 80.22 & 48.24 \\
\hline Eragrostis tenella & Grass & 66.22 & 21.70 & 80.15 & 32.68 \\
\hline Biden pilosa & Broad leaves & 47.33 & 24.31 & 80.15 & 20.52 \\
\hline Urena lobata & Broad leaves & 48.44 & 27.84 & 79.93 & 16.60 \\
\hline Vernonia cinerea & Broad leaves & 58.44 & 29.67 & 79.33 & 59.61 \\
\hline Sida acuta & Broad leaves & 69.63 & 26.54 & 79.19 & 38.43 \\
\hline Stachytarpheta jamaicensis & Broad leaves & 3.41 & 28.76 & 78.96 & 46.93 \\
\hline
\end{tabular}




\begin{tabular}{|c|c|c|c|c|c|}
\hline Coctus speciousus & Broad leaves & 48.89 & 23.53 & 78.81 & 48.37 \\
\hline Lantana camara & Broad leaves & 48.07 & 25.10 & 78.74 & 46.93 \\
\hline Panicum maximum & Grass & 56.81 & 25.49 & 78.74 & 32.29 \\
\hline Pennisetum purpureum & Grass & 56.67 & 18.95 & 78.00 & 38.04 \\
\hline Manihot esculenta & Broad leaves & 53.63 & 3.40 & 76.89 & 36.73 \\
\hline Impatiens balsamina & Broad leaves & 54.52 & 20.65 & 76.59 & 40.26 \\
\hline Ottochloa nodosa & Grass & 62.30 & 28.10 & 76.00 & 32.55 \\
\hline Crassocephalum crepidioides & Broad leaves & 44.37 & -10.98 & 75.78 & 30.59 \\
\hline Imperata cylindrica & Grass & 47.70 & 13.07 & 75.63 & 38.17 \\
\hline Lycopediella cernua & Fern & 60.37 & 49.15 & 75.56 & 67.45 \\
\hline Antigonon leptopus & Broad leaves & 49.85 & 18.95 & 75.48 & 44.44 \\
\hline Leptochloa chinensis & Grass & 53.04 & 15.56 & 75.11 & 36.21 \\
\hline Pyrossia lanceolata & Fern & 26.59 & -2.48 & 75.04 & 42.61 \\
\hline Eleusine indica & Grass & 42.37 & -8.63 & 74.22 & 32.55 \\
\hline Nephrolepis biserrata & Pakis & 50.37 & 39.61 & 73.48 & 45.75 \\
\hline Boerhavia diffusa & Broad leaves & 56.00 & -43.53 & 73.33 & -13.33 \\
\hline Blumea lacera & Broad leaves & 36.81 & 3.14 & 73.19 & 37.91 \\
\hline Pteris vittata & Fern & 46.89 & 8.24 & 72.81 & 27.71 \\
\hline Ischaemum muticum & Grass & 48.37 & 12.03 & 72.15 & 28.63 \\
\hline Echinodorus palaefolius & Broad leaves & 58.81 & 33.86 & 72.00 & 44.71 \\
\hline Chrysopogon aciculatus & Grass & 54.96 & 28.37 & 71.78 & 44.31 \\
\hline Emilia sonchifolia & Broad leaves & 48.44 & 6.54 & 71.70 & 43.27 \\
\hline Stenochlaena palustris & Fern & 33.70 & -14.38 & 71.63 & 15.50 \\
\hline Cyperus iria & Sedge & 43.04 & 20.65 & 71.48 & 36.21 \\
\hline Euphorbia hirta & Broad leaves & 37.93 & -33.86 & 71.19 & -5.10 \\
\hline Tatracera scandens & Broad leaves & 39.27 & 20.29 & 70.37 & 40.78 \\
\hline Davallia denticulata & Fern & 40.22 & 0.52 & 70.37 & 22.09 \\
\hline Brachiaria mutica & Grass & 39.27 & 20.59 & 69.93 & 29.67 \\
\hline Mikania micrantha & Broad leaves & 39.26 & 0.00 & 69.63 & 38.30 \\
\hline Spigelia anthelmia & Fern & 45.48 & -4.58 & 69.26 & 17.12 \\
\hline Cheilanthes tenuifolia & Fern & 34.74 & 30.07 & 69.19 & 46.93 \\
\hline Celosia argentea & Broad leaves & 38.07 & -15.95 & 68.37 & 14.64 \\
\hline Cyperus kyllingia & Sedge & 47.11 & 13.07 & 68.37 & 37.91 \\
\hline Physalis minima & Broad leaves & 39.26 & 0.00 & 67.85 & 35.29 \\
\hline Axonopus compresus & Grass & 47.56 & 3.14 & 66.67 & 18.95 \\
\hline Taenitis blechnoides & Fern & 34.37 & 9.02 & 66.22 & 25.23 \\
\hline Cyperus rotundus & Sedge & 46.67 & 12.68 & 64.96 & 13.33 \\
\hline Rhynchospora corymbosa & Sedge & 42.67 & 17.39 & 64.81 & 26.93 \\
\hline Scirpus grossus & Sedge & 29.93 & 5.62 & 63.78 & 30.94 \\
\hline Bothriochloa bladhii & Grass & 36.07 & 1.24 & 63.78 & 44.97 \\
\hline Cassia alata & Broad leaves & 41.93 & 25.88 & 62.37 & 36.21 \\
\hline Alpina galanga & Broad leaves & 37.11 & 17.65 & 60.89 & 32.81 \\
\hline Centella asiatica & Broad leaves & 40.22 & 0.52 & 60.59 & 9.84 \\
\hline Dillenia excelsa & Broad leaves & 56.52 & 19.74 & 59.63 & 16.37 \\
\hline Paspalum conjugatum & Grass & 35.78 & 1.96 & 53.85 & 7.54 \\
\hline Fimbristylis globulosa & Sedge & 37.48 & 18.43 & 51.27 & 12.37 \\
\hline Vittaria ensiformis & Fern & 34.00 & -1.96 & 42.89 & 23.40 \\
\hline Christella dentate & Fern & 15.33 & -11.50 & 41.04 & 19.61 \\
\hline Lygodium flexuosum & Fern & 19.26 & 4.58 & 34.00 & 0.39 \\
\hline $\operatorname{Min}(M)$ & & 53.55 & 20.04 & 77.17 & 43.54 \\
\hline Standard deviation (i) & & 15.93 & 19.74 & 11.59 & 21.34 \\
\hline *** $100-((\mathrm{M}-1.5(\mathrm{i}))$ & & & & 94.55 & 75.55 \\
\hline ** 100-((M-1.0(i)) & & & & 88.75 & 64.88 \\
\hline * $\quad 100-((\mathrm{M}-0.5(\mathrm{i}))$ & & & & 82.96 & 54.21 \\
\hline
\end{tabular}

${ }^{*}$ Criterion $\left.\left({ }^{*}\right),{ }^{* *}\right)$ and $\left({ }^{* *}\right)$ show a higher percentage of growth inhibition on radicle and hypocotyl of lettuce seedlings compared to controls, $50 \mathrm{mg}$ leaf litter debris was used in the sandwich method for criterion assessment due to its high inhibition percentage on the radicle and hypocotyl growth of $L$. sativa rather than $10 \mathrm{mg}$ leaf litter debris 
Table 3: Allelopathic activity (\% inhibition of lettuce radicle growth) based on 100 common weed species found in Malaysia using dish pack method

\begin{tabular}{|c|c|c|c|c|c|}
\hline \multirow[t]{3}{*}{ Species name } & \multirow{3}{*}{$\begin{array}{l}\text { Morphological } \\
\text { characteristics }\end{array}$} & \multicolumn{4}{|c|}{ Distances } \\
\hline & & $41 \mathrm{~mm}$ & $58 \mathrm{~mm}$ & $82 \mathrm{~mm}$ & $92 \mathrm{~mm}$ \\
\hline & & \multicolumn{4}{|c|}{ Radicle inhibition percentage $(\% \pm)$} \\
\hline Passiflora foetida & Broad leaves & 100.00 & 100.00 & 100.00 & 100.00 \\
\hline Clinacanthus nutans & Broad leaves & 94.06 & 89.66 & 76.63 & 71.26 \\
\hline Tetracera scandens & Grass & 89.85 & 44.06 & 27.59 & 26.82 \\
\hline Ischaemum muticum & Grass & 84.87 & 40.61 & 35.25 & 36.78 \\
\hline Digitaria setigera & Grass & 78.16 & 80.84 & 75.86 & 75.86 \\
\hline Stenochlaena palustris & Fern & 68.20 & 45.59 & 57.09 & 45.21 \\
\hline Pyrossia lanceolata & Fern & 68.01 & 35.63 & 55.56 & 47.51 \\
\hline Blechnum finlaysonianum & Fern & 67.05 & 42.53 & 35.25 & 38.70 \\
\hline Vittaria ensiformis & Fern & 65.90 & 56.70 & 46.74 & 47.51 \\
\hline Manihot esculenta & Broad leaves & 62.84 & 59.00 & 60.92 & 61.30 \\
\hline Cheilanthes tenuifolia & Fern & 62.24 & 60.15 & 31.42 & 30.65 \\
\hline Adiantum latifolium & Fern & 61.11 & 42.91 & 49.43 & 41.38 \\
\hline Lygodium flexuosum & Fern & 59.77 & 47.89 & 49.43 & 55.94 \\
\hline Microsorum pustulatum & Fern & 59.77 & 46.74 & 40.61 & 34.48 \\
\hline Christella dentata & Fern & 58.81 & 49.81 & 48.28 & 41.00 \\
\hline Sporobolus diander & Grass & 56.32 & 46.74 & 46.74 & 45.21 \\
\hline Amaranthus viridis & Broad leaves & 55.94 & 52.11 & 52.11 & 49.81 \\
\hline Pteris vittata & Fern & 55.94 & 43.30 & 27.20 & 8.81 \\
\hline Ipomoea cairica & Broad leaves & 55.17 & 52.87 & 27.97 & 47.13 \\
\hline Triumfetta repens & Broad leaves & 54.79 & 53.64 & 54.79 & 47.89 \\
\hline Senna obtusifolia & Broad leaves & 53.26 & 48.28 & 51.72 & 50.57 \\
\hline Echinochloa colona & Grass & 52.68 & 48.28 & 43.68 & 40.61 \\
\hline Imperata cylindrica & Grass & 52.30 & 48.66 & 55.56 & 47.51 \\
\hline Lycopediella cernua & Fern & 51.92 & 41.00 & 25.29 & 32.18 \\
\hline Alpina galanga & Broad leaves & 51.72 & 52.49 & 51.72 & 54.41 \\
\hline Celosia argentea & Broad leaves & 51.34 & 48.28 & 48.28 & 55.17 \\
\hline Vernonia cinerea & Broad leaves & 50.77 & 51.34 & 43.68 & 57.85 \\
\hline Davallia denticulata & Fern & 50.77 & 38.70 & 47.89 & 32.95 \\
\hline Pistia stratiotes & Broad leaves & 50.38 & 47.89 & 43.30 & 51.34 \\
\hline Axonopus compresus & Grass & 49.43 & 37.16 & 39.46 & 37.16 \\
\hline Rhynchelytrum repens & Grass & 47.51 & 42.53 & 38.70 & 40.23 \\
\hline Crassocephalum crepidioides & Broad leaves & 47.13 & 42.91 & 50.57 & 51.34 \\
\hline Isotoma longiflora & Broad leaves & 46.74 & 50.57 & 49.04 & 41.00 \\
\hline Spigelia anthelmia & Broad leaves & 46.17 & 53.26 & 46.74 & 50.96 \\
\hline Nephrolepis biserrata & Fern & 45.02 & 33.33 & 41.76 & 26.05 \\
\hline Asplenium nidus & Fern & 43.68 & 34.10 & 42.15 & 26.44 \\
\hline Tridax procumbens & Broad leaves & 41.76 & 44.44 & 45.21 & 45.59 \\
\hline Alocasia macrorrhiza & Broad leaves & 39.85 & 24.90 & 45.98 & 52.87 \\
\hline Taenitis blechnoides & Fern & 39.46 & 45.21 & 37.93 & 21.46 \\
\hline Asystasia gangentica & Broad leaves & 38.89 & 21.46 & 24.52 & 36.78 \\
\hline Clidemia hirta & Broad leaves & 38.70 & 17.24 & 22.22 & 18.01 \\
\hline Brachiaria mutica & Grass & 37.93 & 33.33 & 27.59 & 32.18 \\
\hline Sporobolus indicus & Grass & 37.93 & 32.95 & 32.57 & 35.63 \\
\hline Dicranopteris linearis & Fern & 37.16 & 34.87 & 36.02 & 29.50 \\
\hline Neptunia oleracea & Broad leaves & 36.97 & 16.86 & 27.97 & 22.22 \\
\hline Boereria latifolia & Broad leaves & 36.59 & 40.23 & 32.57 & 33.72 \\
\hline Blumea lacera & Broad leaves & 35.82 & 24.14 & 20.69 & 18.01 \\
\hline Leptochloa chinensis & Grass & 34.87 & 33.33 & 21.84 & 26.44 \\
\hline Stachytarphetajamaicensis & Broad leaves & 34.67 & 39.08 & 26.82 & 32.57 \\
\hline Ottochloa nodosa & Grass & 34.29 & 21.46 & 34.48 & 36.02 \\
\hline Chromolaena odorata & Broad leaves & 32.38 & 19.54 & 29.50 & 28.74 \\
\hline
\end{tabular}




\begin{tabular}{|c|c|c|c|c|c|}
\hline Mikania micrantha & Broad leaves & 32.38 & 21.46 & 30.27 & 39.85 \\
\hline Zehneria marginata & Broad leaves & 31.99 & 25.29 & 52.49 & 50.19 \\
\hline Polygonum barbatum & Grass & 31.99 & 34.48 & 30.65 & 29.89 \\
\hline Centrosema pubescens & Broad leaves & 31.80 & 29.89 & 32.57 & 38.31 \\
\hline Scirpus grossus & Sedge & 31.42 & 23.37 & 10.73 & 19.54 \\
\hline Emilia sonchifolia & Broad leaves & 31.23 & 19.54 & 26.44 & 24.57 \\
\hline Eragrostis tenella & Grass & 31.23 & 27.59 & 29.12 & 34.10 \\
\hline Impatiens balsamina & Broad leaves & 30.84 & 22.99 & 24.90 & 27.20 \\
\hline Eleusine indica & Grass & 30.84 & 28.74 & 22.99 & 28.74 \\
\hline Fimbristylis globulosa & Sedge & 30.84 & 25.67 & 30.27 & 31.42 \\
\hline Urena lobata & Broad leaves & 30.08 & 34.10 & 45.59 & 53.64 \\
\hline Cyperus kyllingia & Sedge & 30.08 & 24.14 & 22.22 & 18.01 \\
\hline Eragrostis atrovirens & Grass & 29.89 & 25.67 & 20.69 & 29.12 \\
\hline Boerhavia diffusa & Broad leaves & 29.69 & 27.59 & 49.81 & 39.85 \\
\hline Calopogonium mucunoides & Broad leaves & 29.31 & 33.33 & 36.78 & 24.14 \\
\hline Solanum torvum & Broad leaves & 28.16 & 18.01 & 29.12 & 32.95 \\
\hline Cassia alata & Broad leaves & 27.97 & 27.20 & 31.42 & 40.23 \\
\hline Antigonon leptopus & Broad leaves & 27.39 & 24.90 & 31.42 & 28.74 \\
\hline Cyperus rotundus & Sedge & 27.20 & 24.90 & 21.84 & 32.57 \\
\hline Eliptica alba & Broad leaves & 26.82 & 27.97 & 18.39 & 18.39 \\
\hline Coctus speciousus & Broad leaves & 26.25 & 22.61 & 40.23 & 22.61 \\
\hline Andrographis paniculata & Broad leaves & 25.86 & 26.05 & 26.05 & 17.24 \\
\hline Panicum maximum & Grass & 25.48 & 14.94 & 23.37 & 22.61 \\
\hline Cyperus iria & Sedge & 25.29 & 25.29 & 21.07 & 14.56 \\
\hline Rhynchospora corymbosa & Sedge & 25.29 & 26.44 & 31.80 & 17.62 \\
\hline Zornia diphylla & Broad leaves & 25.19 & 13.79 & 14.56 & 23.37 \\
\hline Lantana camara & Broad leaves & 25.10 & 22.22 & 23.37 & 15.71 \\
\hline Fimbristylis miliacea & Sedge & 24.90 & 20.31 & 22.22 & 22.61 \\
\hline Sida acuta & Broad leaves & 24.71 & 20.31 & 11.88 & 5.75 \\
\hline Melastoma malabathricum & Broad leaves & 24.33 & 27.59 & 34.87 & 31.80 \\
\hline Dactylotenium aegyptium & Grass & 24.14 & 22.99 & 20.69 & 30.27 \\
\hline Chloris barbata & Grass & 24.14 & 19.92 & 29.50 & 32.18 \\
\hline Croton hirtus & Broad leaves & 23.95 & 15.33 & 22.22 & 14.94 \\
\hline Bothriochloa bladhii & Grass & 23.75 & 17.24 & 26.44 & 19.92 \\
\hline Phyllanthus niruri & Broad leaves & 23.56 & 5.75 & 21.46 & 17.24 \\
\hline Ageratum conyzoides & Broad leaves & 23.18 & 13.03 & 27.97 & 19.16 \\
\hline Echinodorus palaefolius & Broad leaves & 22.03 & 18.01 & 22.99 & 24.52 \\
\hline Pennisetum purpereum & Grass & 21.46 & 21.84 & 16.09 & 22.61 \\
\hline Cynodon dactylon & Grass & 21.46 & 26.05 & 13.03 & 24.14 \\
\hline Centella asiatica & Broad leaves & 21.07 & 16.86 & 23.75 & 14.18 \\
\hline Physalis minima & Broad leaves & 20.11 & 23.37 & 13.79 & 19.16 \\
\hline Euphorbia hirta & Broad leaves & 20.11 & 18.77 & 15.33 & 11.11 \\
\hline Paspalum conjugatum & Grass & 19.92 & 12.26 & 22.99 & 16.48 \\
\hline Hedyotis verticillata & Broad leaves & 18.58 & 25.29 & 11.49 & 4.98 \\
\hline Pipersarmentosum & Broad leaves & 17.24 & 16.09 & 15.33 & 8.81 \\
\hline Dillenia excelsa & Broad leaves & 17.05 & 19.16 & 16.86 & 16.48 \\
\hline Biden pilosa & Broad leaves & 16.48 & 13.03 & 14.94 & 11.49 \\
\hline Chrysopogon aciculatus & Grass & 16.09 & 18.01 & 29.12 & 42.53 \\
\hline Momordica balsamina & Broad leaves & 8.62 & 19.16 & 8.81 & 15.71 \\
\hline $\operatorname{Min}(M)$ & & 39.43 & 33.47 & 34.04 & 33.20 \\
\hline Standard deviation (i) & & 18.16 & 16.25 & 15.41 & 15.85 \\
\hline * $\quad 100-((M-0.5(i))$ & & & & & \\
\hline ** $100-((\mathrm{M}-1.0(\mathrm{i}))$ & & & & & \\
\hline *** 100-((M-1.5(i)) & & & & & \\
\hline
\end{tabular}

${ }^{*}$ Criterion $\left({ }^{*}\right),\left({ }^{* *}\right)$ and $\left({ }^{* *}\right)$ show a higher percentage of radicle growth inhibition of lettuce seedlings compared with controls, 41 mm position distance used in the dish pack method for criterion assessment due to its higher inhibitory activity on the radicle growth of $L$. sativa 
Table 4 : Allelopathic activity (\% inhibition of lettuce hypocotyl growth) based on 100 common weed species found in Malaysia using dish pack method

\begin{tabular}{|c|c|c|c|c|c|}
\hline \multirow[t]{3}{*}{ Species name } & \multirow{3}{*}{$\begin{array}{l}\text { Morphological } \\
\text { characteristics }\end{array}$} & \multicolumn{3}{|c|}{ Distances } & \multirow{3}{*}{$92 \mathrm{~mm}$} \\
\hline & & $41 \mathrm{~mm}$ & $58 \mathrm{~mm}$ & $82 \mathrm{~mm}$ & \\
\hline & & \multicolumn{3}{|c|}{ Hypocotyl inhibition percentage ( $\% \pm$ ) } & \\
\hline Passiflora foetida & Broad leaves & 100.00 & 100.00 & 100.00 & 100.00 \\
\hline Digitaria setigera & Grass & 72.55 & 77.78 & 73.20 & 69.93 \\
\hline Clinacanthus nutans & Broad leaves & 71.57 & 67.32 & 43.14 & 41.18 \\
\hline Tetracera scandens & Broad leaves & 69.93 & 7.84 & 4.58 & 0.00 \\
\hline Vittaria ensiformis & Fern & 69.61 & 49.02 & 43.14 & 35.95 \\
\hline Christella dentata & Fern & 60.78 & 49.67 & 41.83 & 41.18 \\
\hline Staechnoclaena palustris & Fern & 60.46 & 50.33 & 30.72 & 41.18 \\
\hline Blechnum finlaysonianum & Fern & 55.56 & 23.53 & 30.72 & 7.84 \\
\hline Manihot esculenta & Broad leaves & 53.27 & 55.56 & 54.25 & 52.94 \\
\hline Lygodium flexuosum & Fern & 52.61 & 40.52 & 30.07 & 44.44 \\
\hline Microsorum pustulatum & Fern & 51.63 & 42.48 & 33.33 & 37.25 \\
\hline Ischaemum muticum & Grass & 50.98 & 7.84 & -3.92 & -3.27 \\
\hline Pyrossia lanceolata & Fern & 50.65 & 35.95 & 41.18 & 44.44 \\
\hline Cheilanthes tenuifolia & Fern & 36.93 & 32.03 & 32.68 & 26.14 \\
\hline Ipomoea cairica & Broad leaves & 35.95 & 33.99 & 32.68 & 34.64 \\
\hline Adiantum latifolium & Fern & 32.68 & 26.14 & 31.37 & 24.18 \\
\hline Senna obtusifolia & Broad leaves & 31.37 & 20.26 & 24.84 & 16.99 \\
\hline Piper sarmentosum & Broad leaves & 31.05 & 28.76 & 18.30 & 5.88 \\
\hline Sporobolus diander & Grass & 31.05 & 30.07 & 30.07 & 22.88 \\
\hline Imperata cylindrica & Grass & 30.16 & 27.45 & 29.41 & 22.22 \\
\hline Amaranthus viridis & Broad leaves & 29.74 & 33.33 & 26.80 & 22.22 \\
\hline Boerhavia diffusa & Broad leaves & 28.76 & 23.53 & 23.53 & 20.26 \\
\hline Urenalobata & Broad leaves & 25.82 & 25.49 & 20.26 & 20.92 \\
\hline Pteris vittata & Fern & 25.49 & 15.69 & 9.80 & 5.23 \\
\hline Triumfetta repens & Broad leaves & 25.16 & 27.45 & 28.10 & 20.92 \\
\hline Zehneria marginata & Broad leaves & 23.86 & 26.80 & 24.84 & 22.22 \\
\hline Pistia stratiotes & Broad leaves & 23.86 & 18.95 & 18.95 & 27.45 \\
\hline Spigelia anthelmia & Broad leaves & 23.53 & 27.45 & 22.88 & 23.53 \\
\hline Asplenium nidus & Fern & 23.53 & 39.22 & 11.11 & 12,42 \\
\hline Melastoma malabathricum & Broad leaves & 23.20 & 16.99 & 21.57 & 17.65 \\
\hline Neptunia oleracea & Broad leaves & 22.22 & 14.38 & 14.38 & 16.34 \\
\hline Rhynchelytrum repens & Grass & 22.22 & 15.69 & 17.65 & 16.99 \\
\hline Emilia sonchifolia & Broad leaves & 20.26 & 11.11 & 11.76 & 16.99 \\
\hline Lycopediella cernua & Fern & 20.26 & 20.26 & 15.03 & 5.88 \\
\hline Vernonia cinerea & Broad leaves & 19.85 & 16.18 & 15.44 & 13.97 \\
\hline Taenitis blechnoides & Fern & 19.28 & 18.95 & -13.73 & -3.27 \\
\hline Mikania micrantha & Broad leaves & 18.63 & 22.22 & 18.95 & 17.65 \\
\hline Crassocephalum crepidioides & Broad leaves & 18.30 & 18.95 & 16.34 & 16.99 \\
\hline Isotoma longiflora & Broad leaves & 17.94 & 17.06 & 12.35 & 13.53 \\
\hline Leptochloa chinensis & Grass & 17.65 & 11.11 & 0.00 & 3.27 \\
\hline Alpina galanga & Broad leaves & 17.32 & 21.57 & 19.61 & 25.49 \\
\hline Clidemia hirta & Broad leaves & 16.67 & 25.49 & 10.46 & 11.76 \\
\hline Echinochloa colona & Grass & 15.36 & 13.73 & 22.22 & 14.38 \\
\hline Asystasiagangentica & Broad leaves & 14.05 & 22.22 & 11.76 & 11.76 \\
\hline Dicranopteris linearis & Fern & 13.73 & 7.84 & 2.61 & -6.54 \\
\hline Ageratum conyzoides & Broad leaves & 13.07 & 21.57 & 21.57 & 15.03 \\
\hline Tridax procumbens & Broad leaves & 13.07 & 9.8 & 16.99 & 8.50 \\
\hline Celosia argentea & Broad leaves & 13.04 & 16.49 & 16.27 & 10.97 \\
\hline Alocasia macrorrhiza & Broad leaves & 12.92 & 16.43 & 12.92 & 11.24 \\
\hline Eragrostis atrovirens & Grass & 12.09 & 12.42 & 8.5 & 20.26 \\
\hline
\end{tabular}




\begin{tabular}{|c|c|c|c|c|c|}
\hline Chromolaena odorata & Broad leaves & 11.44 & 3.27 & 14.38 & -1.96 \\
\hline Cyperus kyllingia & Sedge & 11.44 & 4.58 & 10.46 & 10.46 \\
\hline Ottochloa nodosa & Grass & 11.44 & 7.19 & 5.88 & 13.07 \\
\hline Euphorbia hirta & Broad leaves & 10.78 & 1.31 & -3.92 & -10.46 \\
\hline Lantana camara & Broad leaves & 9.48 & 9.8 & 5.23 & -0.65 \\
\hline Eragrostis tenella & Grass & 9.48 & -2.61 & 0.65 & -1.31 \\
\hline Rhynchospora corymbosa & Sedge & 9.15 & 11.11 & 6.54 & -3.27 \\
\hline Brachiaria mutica & Grass & 8.82 & 7.19 & 7.19 & 10.46 \\
\hline Cyperus rotundus & Sedge & 7.84 & 7.19 & 15.69 & 13.07 \\
\hline Panicum maximum & Grass & 7.52 & 3.27 & 14.38 & 13.73 \\
\hline Boereria latifolia & Broad leaves & 7.19 & 16.34 & 7.19 & 9.8 \\
\hline Fimbristylis globulosa & Sedge & 6.86 & 5.23 & 10.46 & -0.65 \\
\hline Eliptica alba & Broad leaves & 6.21 & -4.58 & -5.88 & -3.27 \\
\hline Coctus speciousus & Broad leaves & 5.56 & 3.92 & 11.11 & 4.58 \\
\hline Axonopus compresus & Grass & 5.56 & 4.58 & 9.15 & 3.92 \\
\hline Antigonon leptopus & Broad leaves & 4.90 & 5.88 & 7.19 & 5.23 \\
\hline Blumea lacera & Broad leaves & 4.58 & 4.88 & 3.27 & -4.58 \\
\hline Cassia alata & Broad leaves & 4.25 & 9.8 & 7.19 & 7.84 \\
\hline Scirpus grossus & Sedge & 3.59 & -1.96 & -19.61 & -1.31 \\
\hline Croton hirtus & Broad leaves & 2.61 & -2.61 & 14.38 & 0.00 \\
\hline Polygonum barbatum & Grass & 2.61 & 8.50 & 2.61 & 8.5 \\
\hline Nephrolepis biserrata & Fern & 2.29 & 1.31 & 10.46 & -3.27 \\
\hline Fimbristylis miliacea & Sedge & 2.21 & 4.41 & 11.03 & 3.68 \\
\hline Impatiens balsamina & Broad leaves & 1.96 & 1.96 & 1.31 & -2.61 \\
\hline Chrysopogon aciculatus & Grass & 1.96 & 1.96 & 3.92 & 3.27 \\
\hline Sporobolus indicus & Grass & 1.31 & 6.54 & 0.65 & 1.96 \\
\hline Cyperus iria & Sedge & 0.65 & 7.19 & -0.65 & 3.92 \\
\hline Phyllanthus niruri & Broad leaves & 0.33 & -13.07 & -3.27 & -9.15 \\
\hline Andrographis paniculata & Broad leaves & 0.33 & -1.96 & 8.5 & -5.23 \\
\hline Centella asiatica & Broad leaves & 0.00 & 1.96 & 9.8 & 5.88 \\
\hline Chloris barbata & Grass & -0.33 & 0.00 & -0.65 & 1.31 \\
\hline Calopogonium mucunoides & Broad leaves & -0.65 & -4.58 & 13.07 & 5.88 \\
\hline Cynodon dactylon & Grass & -0.98 & 1.96 & -5.88 & -5.88 \\
\hline Bothriochloa bladhii & Grass & -1.31 & -0.65 & -3.27 & -5.23 \\
\hline Eleusine indica & Grass & -1.63 & 7.19 & 0.65 & 3.92 \\
\hline Biden pilosa & Broad leaves & -2.61 & -7.19 & -9.15 & -458 \\
\hline Davallia denticulata & Fern & -2.61 & 9.15 & 5.88 & -1.31 \\
\hline Physalis minima & Broad leaves & -4.25 & -1.96 & -3.23 & -3.78 \\
\hline Echinodorus palaefolius & Broad leaves & -4.58 & -2.61 & -8.50 & -3.27 \\
\hline Pennisetum purpereum & Grass & -4.90 & -4.58 & -10.46 & -2.61 \\
\hline Dactylotenium aegyptium & Grass & -5.23 & 2.61 & 4.48 & 3.92 \\
\hline Stachytarpheta jamaicensis & Broad leaves & -6.54 & 5.23 & -6.54 & 1.96 \\
\hline Paspalum conjugatum & Grass & -7.84 & -6.54 & -3.27 & -12.42 \\
\hline Solanum torvum & Broad leaves & -10.13 & -13.07 & -8.50 & -7.84 \\
\hline Zornia diphylla & Broad leaves & -12.09 & -11.76 & -10.46 & -8.50 \\
\hline Sida acuta & Broad leaves & -13.73 & -9.80 & -16.34 & -18.30 \\
\hline Dillenia excelsa & Broad leaves & -15.36 & -19.61 & -11.11 & -11.11 \\
\hline Hedyotis verticillata & Broad leaves & -21.24 & -19.61 & -24.18 & -24.18 \\
\hline Momordica balsamina & Broad leaves & -21.57 & -32.68 & -13.07 & -16.34 \\
\hline $\operatorname{Min}(M)$ & & 16.81 & & & \\
\hline Standard deviation (i) & & 22.25 & & & \\
\hline * $\quad 100-((M-0.5(\mathrm{i}))$ & & & & & \\
\hline ** 100-((M-1.0(i)) & & & & & \\
\hline *** 100-((M-1.5(i)) & & & & & \\
\hline
\end{tabular}

${ }^{*}$ Criterion $\left({ }^{*}\right),\left({ }^{* *}\right)$ and $\left({ }^{* * *}\right)$ show a higher percentage of hypocotyl growth inhibition of lettuce seedlings compared with controls, $41 \mathrm{~mm}$ position distance used in the dish pack method for criterion assessment due to its higher inhibitory activity on the hypocotyl growth of $L$. sativa 
with $200 \mathrm{mg}$ of leaf litter, P. foetida, Clinacanthus nutans, Tetracera scandens and Ischaemum muticum showed $80 \%$ inhibition in lettuce radicle. It is noteworthy that some weed species ( $88 \%$ of species) decreased the growth of lettuce seedlings with increasing distances from the source well (from 92 $\mathrm{mm}, 82 \mathrm{~mm}, 58 \mathrm{~mm}$ to $41 \mathrm{~mm}$ ). The results of this study are in line with the study by Appiah et al. (2015) who found that several plant species in Sino-Japanese region like Photinia glabra, Liquidambar styraciflua and Cinnamomum camphora inhibited the growth of lettuce radicle by dish pack method. Similarly, Nurul Ain et al. (2017) found that 15 common weed species in Malaysia inhibited the radicle growth of lettuce seedlings by dish pack method at the nearest distance of $41 \mathrm{~mm}$ from the leaf litter of common weed. Based on the effects of weeds leaf volatilization studied, P. foetida showed the highest percent inhibition on lettuce seedling with $100 \%$ inhibition at all distances.

On the basis of results it can be concluded that $P$. foetida weed possessed the highest allelopathic effect than other 99 weed and medicinal plants species studied by sandwich and dish pack method. The ability of $P$. foetida to completely inhibit the radicle and hypoctyl growth of lettuce at all distances tested using dish pack method proved the presence of volatile compound in $P$. foetida leaf extract release of their allelochemical by volatilization. On the other hand, inhibition of radicle and hypocotyl of lettuce was cent percent achieved when $50 \mathrm{mg}$ of $P$. foetida leaf litter was treated by sandwich method and showed that leachate from the leaf extract contributed to the highest allelopathic effect compared to other weed species. This study is a preliminary work of screening some common weeds found in Malaysia that have allelopathic potentials. Subsequently, further studies should be conducted on the identification and effects of allelochemicals secreted by the plants. This effort is important for synthesizing bioactive compounds from weed species as a natural product for controlling weed propagation.

\section{Acknowledgments}

This research was supported by Universiti Kebangsaan Malaysia (UKM) research grant, GUP-2014-087. Thanks, are also extended to the lab assistants and staff of Plant Biotechnology Lab, Greenhouse Complex, Universiti Kebangsaan Malaysia for their assistance and suggestion to improve this article.

\section{Add-on Information}

Authors' contribution: M.S. Ishak: Conceived the presented idea, wrote the first draft; M.B. Nurul Ain: Conducted experiment, conceived the presented idea; I. Sahid: supervised, contributed to final version; K. Mardiana-Jansar: supervised, contributed to final version.

Research content: The research content is original and has not been published elsewhere

\section{Ethical approval: NotApplicable}

Conflict of interest: The authors declare that there is no conflict of interest.

\section{Data from other sources: NotApplicable}

Consent to publish: All authors agree to publish the paper in Journal of Environmental Biology.

\section{References}

Aktar, M.W., D. Sengupta and A. Chowdury: Impact of pesticides use in agriculture: Their benefits and hazards. Interdiscip. Toxicol., 2, 1$12(2009)$.

Ali, K.W., M.I. Shinwari and S. Khan: Screening of 196 medicinal plant species leaf litter for allelopathic potential. Pak. J. Bot., 51, 21692177 (2019).

Appiah, K.S., Z. Li, R. Zeng, S. Luo, Y. Oikawa and Y. Fujii: Determination of allelopathic potentials in plant species in Sino-Japanese floristic region by sandwch method and dish pack method. IJBAS, 4, 381394 (2015).

Assaduzzaman, M., S. Shamima and M.I. Mahbub: Allelopathy and allelochemicals in rice weed management. Ban. Res. Public. J., 4, 1-14 (2010).

Chick, T.A. and J.J. Keilbaso: Allelopathy as an inhibition factor in ornamental tree growth: Implication from the literature. Arboric. J., 24, 274-279(1998).

Chou, C.H.: Roles of allelopathy in plant biodiversity and sustainable agriculture. CRC Crit. Rev. Plant Sci.,18, 609-636 (1999).

Daehler, C.C., J.S. Denslow, S. Asari and H. Kuo: A risk assessment system for screening out invasive pest plants from Hawaii and other Pacific Islands. Conserv. Biol.,18, 360-368 (2004).

de Albuquerque, M.B., R.C. Santos, M.L. Lima, P. Filho, R.J.M. Nogueira and C.A.G. Camara: Allelopathy, an alternative tool to improve cropping systems: A review. Agron. Sustain. Dev., 31, 279-295 (2010).

Dilipkumar, M., T.S. Chuah, S.S. Goh and I. Sahid: Weed management issues, challenges, and opportunities in Malaysia. Crop Prot., 2017, 1-9 (2017).

Fujii, T., M. Matsuyama, S. Hiradate and H. Shimozawa: Dish pack method: A new bioassay for volatile allelopathy. In: Proceedings of $4^{\text {th }}$ World Congress on Allelopathy, "Establishing the Scientific Base", Fourth World Congress on Allelopathy, Wagga Wagga, New South Wales, Australia (Eds.: J.D.I. Harper, M. An, H. Wu and J.H. Kent). Centre for Rural Social Research, Charles Sturt University, Wagga Wagga, Australia, pp. 493-497 (2005).

Fujii, Y., S.S. Parves, Y. Ohmae and O. lida: Screening of 239 medicinal plant species for allelopathic activity using the sandwich method. Weed Biol. Manag., 3, 233-241 (2003).

Fujii, Y., T. Shibuya, K. Nakatani, T. Itani, S. Hiradate and M.M. Parvez: Assessment method for allelopathic effect from leaf litter leachates. Weed Biol. Manag., 4, 19-23 (2004).

Harper, J.R. and N.E. Balke: Characterization of the inhibition of $\mathrm{K}^{+}$ absorption in oat roots by salicyclic acid. Plant Physiol., 68, 1349$1353(1981)$

Ishak, M.S., B.S. Ismail and N. Yusoff: Allelopathic potential of Leucaena 
leucocephala (Lam.) de Wit on the germination and seedling growth of Ageratum conyzoides L., Tridax procumbens L. and Emilia sonchifolia (L.) DC. Allelopathy J., 37, 109-122 (2016).

Ismail, B.S., P.W. Tan and T.S. Chuah: Assessment of the potential allelopathic effects of Pennisetum purpureum Shumach. on the germination and growth of Eleusine indica (L.) Gaertn. Sains Malays. 44, 269-274 (2015).

Jaishankar, M., T. Tseten, N. Anbalagan, B.B. Mathew and K.N. Beeregowda: Toxicity, mechanism and health effects of some heavy metals. Interdiscip. Toxicol., 7, 60-72 (2014).

Morikawa, C.I.O., R. Miyaura, M.L.T.Y. Figueroa, E.L.R. Salgado and Y. Fujii: Screening of 170 Peruvian plant species for allelopathic activity by using the sandwich method. Weed Biol. Manag., 12, 111 (2012).

Nurul Ain, M.B., Y. Nornasuha and B.S. Ismail: Evaluation of the alleopathic potential of fifteen common Malaysian weeds. Sains Malays., 46, 1413-1420 (2017).

Qin, B., L.G. Perry, C.D. Broeckling, J. Du, F.R. Stermitz, M.W. Paschke and J.M. Vivanco: Phyototoxic allelochemicals from roots and root exudates from Leafy Spurge (Euphorbia esula L.). Plant Signal. Behav., 1, 323-327 (2006).

Reigosa, M.J., A. Sánchez-Moreiras and L. Gonzáles: Ecophysiological approach in allelopathy. CRC Crit. Rev. Plant Sci.,18, 577-608 (1999).

Sahid, I. and K.W. Chan: Integrated ground cover management in plantations. In: Advances in Oil Palm Research. (Eds.: Y. Basiron, B.S. Jalani and K.W. Chan). Malaysian Palm Oil Board, Kuala Lumpur, Malaysia. 1, pp. 623-652 (2000).

Samedani, B., A.S. Juraimi, M.Y. Rafii, S.A. Sheikh Awadz, M.P. Anwar and A.R. Anuar: Effect of cover crops on weed suppression in oil palm plantation. Int. J. Agric. Biol., 17, 251-260 (2015).

Shinwari, M.I. and Y. Fujii: Allelopathic activity of medicinal plants and weeds from Pakistan. Allelopathy J., 32, 223-232 (2013).

Shiraishi, S., I. Watanabe, K. Kuno and Y. Fujii: Allelopathic activity of leaching from dry leaves and exudate from roots of ground cover plants assayed on agar. Weed Biol. Manag., 2, 133-142 (2002). 